\title{
Generalized Bohr-Sommerfeld formula for Schwarzschild black hole normal modes
}

\author{
N Anderssont, M E Araújoł\$ and B F Schutz|| \\ Department of Physics and Astronomy, University of Wales College of Cardiff, \\ Cardiff CF2 3YB, UK
}

Received 14 September 1992

\begin{abstract}
A generalized Bohr-Sommerfeld-type formula determining the quasinormalmode frequencies of Schwarzschild black holes is derived using the phase-integral method with three transition points. The formula is valid provided that these points are well separated from each other, and is remarkably accurate for the high-overtone modes. It makes a great improvement over the conventional Bohr-Sommerfeld formula, derived from two transition points.
\end{abstract}

PACS numbers: $0260,0270,0420,0430,9760 \mathrm{~L}$

\section{Introduction}

Analytic approximation techniques based on complexified versions of the WKB approach have lately proved powerful methods for calculating frequencies of the quasinormal modes of black holes $[1,2]$. We have recently reviewed one of these, the phase integral method, in the context of black hole perturbations [3] to which the reader is referred for notation, conventions and fundamentals of this method. Here we use the phase-integral method to develop a new and more powerful analytic approximation for the low-lying quasinormal modes of Schwarzschild black holes. This method has potential applications to other problems, such as Reissner-Nordström and Kerr.

The first complex-WKB calculations for this problem used a version of the BohrSommerfeld (hereafter BS) formula [1]. It has been shown by Araujo et al [4] and Fröman et al [2] that the BS formula can be derived within the phase-integral method. In this method a key role is played by the transition points, which are the analogues of the WKB turning points. The calculations in [2] and [4] consider only two of the four transition points for this problem. They must be well separated from the other transition points if the derived formulae are to be accurate, although the two points may be close to each other.

\footnotetext{
† E-mail address: spxna@thor.cardiff.ac.uk

‡ E-mail address: araujo@taff.cardiff.ac.uk

$\S$ Permanent address: Departamento de Matemática, Universidade de Brasilia, Caixa Postal 04670, 70.919-000 Brasilia DF, Brazil.

|| E-mail address: schutz@cardiff.ac.uk
} 
The Bs formula is very accurate for the low-lying quasinormal modes, producing results that are in good agreement with the numerical calculations of Leaver [5], Nollert and Schmidt [6] and Andersson [7], all of whom use different methods. However, as the overtone index $n$ increases (see figure 1 in [1]), the four transition points in the complex coordinate plane all tend to approach each other. As a consequence, it is reasonable to expect that the influence of the other two transition points becomes significant, leading to the observed loss of accuracy of the standard BS formula for $n \geqslant 2$ as seen in [1].

The purpose of this paper is to derive an expression for the complex eigenfrequencies of the Schwarzschild black hole quasinormal modes by taking into account three transition points. This expression, which we call the Generalized BohrSommerfeld formula (GBS), is derived using the phase-integral method in the simplest possible context.

The plan of this paper is the following: in section 2 we repeat some of the fundamental formulae for a phase-integral analysis of the problem. Section 3 is devoted to the derivation of the GBS formula. In section 4 we present the numerical results for the quasinormal-mode frequencies calculated from the GBS formula and compare our results with the previously mentioned numerical results of Leaver [5], Nollert and Schmidt [6] and Andersson [7]. Our conclusions are presented in section 5 , where we also make suggestions for further work.

\section{Fundamental formulae}

Assuming a time dependence $\exp (-\mathrm{i} \sigma t)$, small perturbations are governed by the differential equation [3]

$$
\frac{\mathrm{d}^{2} \Psi}{\mathrm{d} r^{2}}+R_{\ell}(r) \Psi=0
$$

For a Schwarzschild black hole the function $R_{\ell}(r)$ is given by

$$
R_{\ell}(r)=\frac{r^{2}}{(r-2)^{2}}\left[\sigma^{2}-V_{\ell}(r)+\frac{2}{r^{3}}-\frac{3}{r^{4}}\right]
$$

and, in the case of gravitational perturbations, the 'effective potential' is

$$
V_{\ell}(r)=\left(1-\frac{2}{r}\right)\left[\frac{\ell(\ell+1)}{r^{2}}-\frac{6}{r^{3}}\right]
$$

where $\ell$ is the spheric harmonical index of the perturbation. In the above formulae all dimensional quantities are scaled with the black hole mass $M$ (This corresponds to setting $M=1$ ). The quasinormal modes are solutions to the differential equation (1) that fulfil the boundary conditions: the waves must be outgoing at spatial infinity and ingoing across the event horizon $(r=2)$.

Since the Wronskian of two exact, linearly independent solutions to (1) must be constant, the general solution can always be expressed as a linear combination of the two functions

$$
f_{1,2}(r)=q^{-1 / 2}(r) \exp \left[ \pm \mathrm{i} \int q(r) \mathrm{d} r\right]=q^{-1 / 2}(r) \exp [ \pm \mathrm{i} w(r)]
$$


where $q(r)$ satisfies the nonlinear equation

$$
q^{1 / 2} \frac{\mathrm{d}^{2}}{\mathrm{~d} r^{2}}\left(q^{-1 / 2}\right)-q^{2}+R_{\ell}=0
$$

The standard WKB approximation corresponds to taking $q \approx R_{\ell}^{1 / 2}$. This approximation is accurate when the derivative in (5) can be neglected, i.e. when $R_{\ell}(r)$ is in some sense slowly varying [8]. In the phase-integral method the function $q(r)$ is approximated at the lowest order by an appropriately chosen function $Q(r)$ such that

$$
q(r) \approx Q(r) \sum_{n=0}^{N} Y_{2 n}
$$

where $Y_{0}$ is equal to unity and the higher-order contributions are given in, for example, [3]. Here it is convenient to take [3]

$$
Q^{2}(r)=R_{\ell}(r)-\frac{1}{4(r-2)^{2}}
$$

in order to prevent the approximation from breaking down close to the horizon of the black hole.

As already mentioned above, the so-called transition points, the zeros of $Q^{2}(r)$, play an important role in a phase-integral analysis. Another important concept is the Stokes and anti-Stokes lines. These are curves along which the quantity $Q(r) \mathrm{d} r$ is either purely imaginary or real, respectively. By imposing the boundary conditions, defining a quasinormal mode, on anti-Stokes lines instead of on the real coordinate axis numerical difficulties can be avoided-see [3] for a comprehensive discussion.

By considering the influence of two (of the four) transition points the Bs formula is obtained [2-4]. It can be written as

$$
\gamma_{21}=\left(n+\frac{1}{2}\right) \pi
$$

where $n$ is a non-negative integer labelling the modes and, in the first order of approximation,

$$
\gamma_{21}=\int_{t_{2}}^{t_{1}} q(r) \mathrm{d} r
$$

The transition points considered in the analysis are $t_{1}$ and $t_{2}$ see figure 1 . In the higher orders of approximation this definition has to be replaced by a contour integral encircling the two transition points-see [3].

\section{Derivation of the generalized Bohr-Sommerfeld formula}

The BS formula generates accurate frequencies for the lowest-lying quasinormal modes. However, its precision deteriorates for modes of overtone index, $n$, higher than two. A possible explanation for this loss of accuracy is the change in the pattern of Stokes and anti-Stokes lines as $n$ increases. We note that for the low-lying modes, 


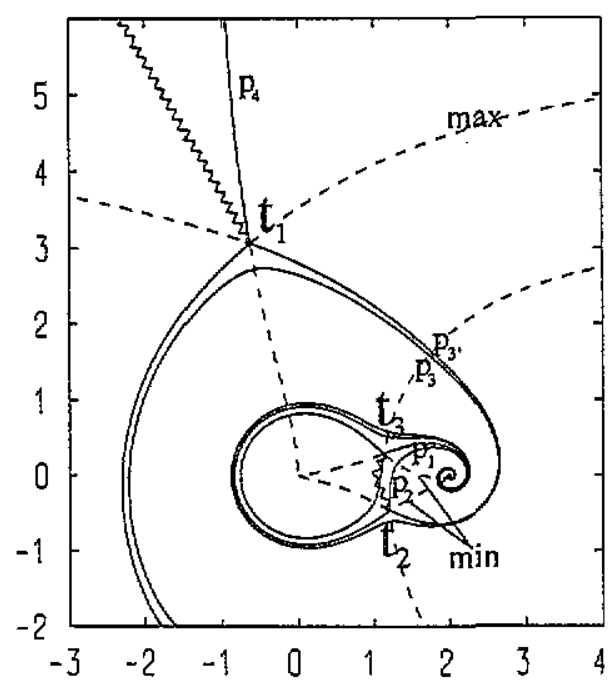

Figure 1. Pattern of Stokes (broken) and anti-Stokes (full) lines emerging from the three transition points under consideration for $n=4$ and $l=2$. The cuts are represented by the zigzagged lines. The labels max and $\min$ on the Stokes lines denote maxima and minima of the function $|\exp (\mathrm{i} w)|$ respectively.

see figure 2 of [3], the anti-Stokes line emanating from $t_{2}$ towards the horizon does not pass close to any other transition point. This is not the case for the higher overtones-see figure 1 of the present paper. This indicates that the assumption that the $F$-matrix connecting the horizon to the point $p_{2}$ is a unit matrix is no longer valid, cf section 3.4 in [3]. It is reasonable to assume that the main reason for the failure of the Bs formula as $n$ increases, is the growing influence of the transition point $t_{3}$. Therefore, $t_{3}$ ought to be considered in an analysis of the highly damped modes.

In order to make $Q(r)$ single-valued we must introduce cuts from each transition point. We choose the cuts as in figure 1 , and we make the choice of phase for $Q(r)$ as in section 3 of [3]. The phase-integral functions $f_{1}(r)$ and $f_{2}(r)$ then represent outgoing waves as $r \rightarrow \infty$ and waves falling across the horizon as $r \rightarrow 2$, respectively. The boundary conditions then require

$$
\Psi \underset{r \rightarrow+2}{\sim} a_{2} f_{2}(r)
$$

and

$$
\Psi \underset{r \rightarrow \infty}{\sim} a_{1} f_{1}(r)
$$

respectively, where $a_{2} \boxminus a_{2}(+2)$ and $a_{1} \equiv a_{1}(\infty)$ are undetermined normalization factors.

We now want to use the phase-integral method to derive an expression for the eigenfunction $\Psi$ at the point $p_{3}$ (figure 1) by continuing the expressions (10) and (11) from the horizon and infinity, respectively. Matching the two expressions obtained yields a formula determining the quasinormal-mode frequencies. 
We impose the boundary condition of outgoing waves falling across the horizon on the spiralling anti-Stokes line that emerges from the transition point $t_{2}$ towards the horizon. Consider the point $p_{1}$ lying on this anti-Stokes line far away from the transition point $t_{3}$ and lying between $t_{3}$ and the horizon. Then, the solution $\Psi$ at $p_{1}$ is

$$
\Psi\left(p_{1}\right)=a_{2} f_{2}\left(p_{1}\right)
$$

since the functional form of this solution does not change when continued along this anti-Stokes line (see expression (25) of [3]). We can continue the solution (12) to the point $p_{2}$, lying on the same anti-Stokes line. In so doing we must circumvent $t_{3}$ along a path that remains far away from it. Any such path crosses a Stokes line on which $\left|\mathrm{e}^{\mathrm{i} w}\right|$ has a minimum. Provided that the two transition points $t_{2}$ and $t_{3}$ are well separated, the $F$-matrix connecting $p_{1}$ and $p_{2}$ is given by expression (32) of [3] and we obtain

$$
\Psi\left(p_{2}\right)=-\mathrm{i} a_{2} f_{1}\left(p_{2}\right)+a_{2} f_{2}\left(p_{2}\right) .
$$

In the expression (13) the transition point $t_{3}$ is used as the reference level for the calculation of $w(r)$ (see expression (24) in [3]). It is convenient for the later matching of the solutions that we switch to $t_{2}$ as the phase reference level. Hence (13) takes the form

$$
\Psi\left(p_{2}\right)=-\mathrm{i} a_{2} \exp \left(\mathrm{i} \gamma_{32}\right) f_{1}\left(p_{2}\right)+a_{2} \exp \left(-\mathrm{i} \gamma_{32}\right) f_{2}\left(p_{2}\right) .
$$

We now want to continue the solution (14) to the point $p_{3}$ lying on the adjacent anti-Stokes lines emanating from $t_{2}$. We use a path that circumvents $t_{2}$ (far away from it) and crosses a Stokes line on which $\left|\mathrm{e}^{\mathrm{i} w}\right|$ has a minimum. The $F$-matrix connecting $p_{2}$ and $p_{3}$ is then given by (27) in [3] and we have that

$$
\Psi\left(p_{3}\right)=-\mathrm{i} a_{2}\left[\exp \left(\mathrm{i} \gamma_{32}\right)+\exp \left(-\mathrm{i} \gamma_{32}\right)\right] f_{1}\left(p_{3}\right)+a_{2} \exp \left(-\mathrm{i} \gamma_{32}\right) f_{2}\left(p_{3}\right) .
$$

Let us now consider the continuation of the solution from infinity to the intended matching point $p_{3}$. We impose the boundary condition of outgoing waves at infinity on the anti-Stokes line emerging from the transition point $t_{1}$ towards infinity. Consider the point $p_{4}$ lying on this anti-Stokes line far away from the transition point $t_{1}$. Then an analogous argument to that leading to (12), yields that the solution $\Psi$ at $p_{4}$ is

$$
\Psi\left(p_{4}\right)=a_{1} f_{1}\left(p_{4}\right) \text {. }
$$

In order to continue the solution (16) to the point $p_{3^{\prime}}$ lying on the adjacent antiStokes line emerging from $t_{1}$ we use a path that circumvents $t_{1}$ while keeping well away from it. This path crosses a Stokes line on which $\left|\mathrm{e}^{\mathrm{i} w}\right|$ has a maximum. The $F$-matrix connecting $p_{4}$ and $p_{3}$, is then given by (26) in [3] and we obtain that

$$
\Psi\left(p_{3^{\prime}}\right)=a_{1} f_{1}\left(p_{3^{\prime}}\right)-\mathrm{i} a_{1} f_{2}\left(p_{3^{\prime}}\right) .
$$

The expression (17) has the transition point $t_{1}$ as the phase reference level for the calculation of $w(r)$. Switching to $t_{2}$ as the reference level yields

$$
\Psi\left(p_{3^{\prime}}\right)=a_{1} \exp \left(-\mathrm{i} \gamma_{21}\right) f_{1}\left(p_{3^{\prime}}\right)-\mathrm{i} a_{1} \exp \left(\mathrm{i} \gamma_{21}\right) f_{2}\left(p_{3^{\prime}}\right) .
$$


Finally, we have to continue the solution to $p_{3}$. This situation is identical to the one analysed in section 3.4 of [3] (see the discussion in connection with expression (53) of that paper). There we show that, provided that $\left|\operatorname{Im} \gamma_{21}\right|$ is small, the $F$-matrix connecting $p_{3}$ to $p_{3^{\prime}}$ is approximately the identity matrix. Hence,

$$
\Psi\left(p_{3}\right)=a_{1} \exp \left(-\mathrm{i} \gamma_{21}\right) f_{1}\left(p_{3}\right)-\mathrm{i} a_{1} \exp \left(\mathrm{i} \gamma_{21}\right) f_{2}\left(p_{3}\right) .
$$

Now, the requirement that expressions (15) and (19) must be identical yields

$$
\begin{aligned}
& a_{1} \exp \left(-\mathrm{i} \gamma_{21}\right)=-\mathrm{i} a_{2}\left[\exp \left(\mathrm{i} \gamma_{32}\right)+\exp \left(-\mathrm{i} \gamma_{32}\right)\right] \\
& -\mathrm{i} a_{1} \exp \left(\mathrm{i} \gamma_{21}\right)=a_{2} \exp \left(-\mathrm{i} \gamma_{32}\right) .
\end{aligned}
$$

Dividing equations (20) by one another gives

$$
\exp \left(-2 \mathbf{i} \gamma_{21}\right)=-\left[1+\exp \left(2 \mathbf{i} \gamma_{32}\right)\right] .
$$

Taking the logarithm of (21) we obtain the generalized Bohr-Sommerfeld formula for the quasinormal modes of Schwarzschild black holes

$$
\gamma_{21}=\left(n+\frac{1}{2}\right) \pi+\frac{1}{2} \mathrm{i} \log \left[1+\exp \left(2 \mathrm{i} \gamma_{32}\right)\right] .
$$

This formula is expected to be valid as long as the pattern of Stokes and antiStokes lines remain similar to that in figure 1 , and all transition points are well separated from each other. A similar formula has previously been discussed, in connection with Regge poles in scattering theory, by Thylwe [9].

\section{Numerical results}

To investigate the accuracy of the GBS formula we have done calculations for the two lowest values of $\ell$. For each quasinormal-mode, with index $n$, calculations were performed for the first five orders of approximation (labelled with odd numbers 1 through 9). The numerical results are given in table $1(\ell=2)$ and table $2(\ell=3)$. In the tables the results obtained from the GBS formula (22) are compared with calculations using the BS formula (8) and with reliable numerical results. For the first ten modes the independent methods of Leaver [5], Nollert and Schmidt [6] and Andersson [7] agree to all figures quoted in our tables. For the remaining high-overtone modes we compare our results with those obtained using the phaseamplitude method of Andersson.

From the tables it follows that the GBS formula is much more accurate than the Bs formula for the high-overtone modes. Generally it yields results with an error in the third decimal place. It is clear, from the numerical results and figure 1, that the main reason for the breakdown of the BS formula is the influence of the third transition point $t_{3}$.

When performing calculations using several different orders of approximation it is necessary to determine which order to consider as the most reliable. It would be very simple if the higher-order contributions were to get smaller as the order of approximation was increased. Since we are integrating an asymptotic series expansion for the function $q(r)$, this is not the case. In a paper by Fröman et al [2] the optimal 
Table 1. Numerical results for $\ell=2$.

\begin{tabular}{|c|c|c|c|c|c|c|c|c|}
\hline \multirow[b]{2}{*}{$n$} & \multicolumn{3}{|c|}{ Bs formula } & \multicolumn{3}{|c|}{ GBs formula } & \multicolumn{2}{|c|}{ Numerical methods } \\
\hline & ord & $\operatorname{Re}(\sigma)$ & $\operatorname{Im}(\sigma)$ & ord & $\operatorname{Re}(\sigma)$ & $\operatorname{Im}(\sigma)$ & $\operatorname{Re}(\sigma)$ & $\operatorname{Im}(\sigma)$ \\
\hline 0 & 3 & 0.3734 & -0.0891 & 3 & 0.3744 & -0.0895 & 0.37367 & -0.08896 \\
\hline 1 & 3 & 0.3452 & -0.2742 & 3 & 0.3492 & -0.2738 & 0.34671 & -0.27391 \\
\hline 2 & 1 & 0.3009 & -0.4855 & 3 & 0.3031 & -0.4749 & 0.30105 & -0.47828 \\
\hline 3 & 1 & 0.2510 & -0.7202 & 3 & 0.2496 & -0.7019 & 0.25150 & -0.70515 \\
\hline 4 & 3 & 0.2022 & -0.9738 & 3 & 0.2049 & -0.9455 & 0.20751 & -0.94684 \\
\hline 5 & 3 & 0.1768 & -1.2324 & 3 & 0.1666 & -1.1947 & 0.16930 & -1.19561 \\
\hline 6 & 3 & 0.1586 & -1.4909 & 3 & 0.1302 & -1.4464 & 0.13325 & -1.44791 \\
\hline 7 & 3 & 0.1450 & -1.7485 & 5 & 0.0921 & -1.7000 & 0.09282 & -1.70384 \\
\hline 9 & 3 & 0.1257 & -2.2610 & 3 & 0.0622 & -2.3117 & 0.06326 & -2.30264 \\
\hline 10 & 5 & 0.1198 & -2.5158 & 3 & 0.0753 & -2.5671 & 0.07655 & -2.56083 \\
\hline 15 & 5 & 0.0961 & -3.7841 & 3 & 0.0865 & -3.8292 & 0.08859 & -3.82511 \\
\hline 20 & 5 & 0.0826 & -5.0455 & 3 & 0.0846 & -5.0854 & 0.08739 & -5.08147 \\
\hline 25 & 5 & 0.0736 & -6.3036 & 3 & 0.0816 & -6.3394 & 0.08497 & -6.33563 \\
\hline 30 & 5 & 0.0670 & -7.5596 & 5 & 0.0789 & -7.5924 & 0.08260 & -7.58868 \\
\hline
\end{tabular}

Table 2. Numerical results for $\ell=3$.

\begin{tabular}{|c|c|c|c|c|c|c|c|c|}
\hline \multirow[b]{2}{*}{$\underline{n}$} & \multicolumn{3}{|c|}{ BS formula } & \multicolumn{3}{|c|}{ GBS formula } & \multicolumn{2}{|c|}{ Numerical methods } \\
\hline & ord & $\operatorname{Re}(\sigma)$ & $\operatorname{Im}(\sigma)$ & ord & $\operatorname{Re}(\sigma)$ & $\operatorname{Im}(\sigma)$ & $\operatorname{Re}(\sigma)$ & $\operatorname{Im}(\sigma)$ \\
\hline 0 & 7 & 0.599445 & -0.092704 & 5 & 0.59947 & -0.09263 & 0.599443 & -0.092703 \\
\hline 1 & 7 & 0.582643 & -0.281305 & 7 & 0.58257 & -0.28085 & 0.582643 & -0.281298 \\
\hline 2 & 5 & 0.55171 & -0.47910 & 5 & 0.5506 & -0.4779 & 0.55168 & -0.47909 \\
\hline 3 & 5 & 0.51202 & -0.69039 & 3 & 0.5079 & -0.6896 & 0.51196 & -0.69034 \\
\hline 4 & 3 & 0.47024 & -0.91545 & 3 & 0.4633 & -0.9200 & 0.47017 & -0.91565 \\
\hline 5 & 3 & 0.4316 & -1.1517 & 3 & 0.4283 & -1.1637 & 0.43139 & -1.15215 \\
\hline 6 & 1 & 0.3974 & -1.3975 & 3 & 0.4027 & -1.4096 & 0.39766 & -1.39591 \\
\hline 7 & 1 & 0.3681 & -1.6458 & 3 & 0.3815 & -1.6548 & 0.36899 & -1.64384 \\
\hline 8 & 1 & 0.3429 & -1.8963 & 1 & 0.3567 & -1.9094 & 0.34462 & -1.89403 \\
\hline 9 & 1 & 0.3208 & -2.1481 & 1 & 0.3389 & -2.1571 & 0.32368 & -2.14540 \\
\hline 10 & 1 & 0.3010 & -2.4009 & 1 & 0.3218 & -2.4055 & 0.30546 & -2.39735 \\
\hline 15 & 1 & 0.2314 & -3.6764 & 1 & 0.2529 & -3.6609 & 0.23925 & -3.65868 \\
\hline 20 & 3 & 0.1847 & -4.9534 & 3 & 0.1907 & -4.9178 & 0.19393 & -4.91832 \\
\hline 25 & 3 & 0.1639 & -6.2202 & 3 & 0.1542 & -6.1754 & 0.15687 & -6.17616 \\
\hline 30 & 3 & 0.1488 & -7.4829 & 3 & 0.1186 & -7.4314 & 0.12147 & -7.43320 \\
\hline
\end{tabular}

order of approximation (used in their tables) corresponds to the most accurate as compared to reliable numerical results. Since this requires the knowledge of results that are believed to be more accurate than those generated using the approximate method, this is a very impractical way of determining which order of approximation to use. If a method is available that gives the accurate results why bother about the phase-integral method at all? In order to make the present method useful in situations where no reliable data are available for comparison we need an independent definition of the optimal order of approximation. We propose that the results obtained in different orders of approximation should be considered as an asymptotic series. To the first-order result is then added consecutively smaller terms until, at a certain order of approximation, the contribution of the next order is larger. We suggest as a simple rule of thumb that this 'series expansion' should be truncated before the smallest term. Theoretically, the smallest contribution can then be considered as an 
estimate of the error in the previous term. Therefore, the optimal order will be that giving rise to the term preceding the smallest term in the series. The reliability of this rule of thumb will be discussed elsewhere.

It should be noted that, for calculations using the BS formula, the optimal order (as defined by the rule of thumb) decreases as the quasinormal-mode index $n$ is increased. This may be used to conclude that the BS formula becomes less reliable. However, the situation is not that simple. When $|\operatorname{Im} \sigma| \gg|\operatorname{Re} \sigma|$ the contributions from the higher orders of approximation again become small (i.e. the optimal order is high). Guinn et al [1] argued that this would imply that the Bs formula gives accurate results. On the other hand, our analysis of the pattern of anti-Stokes lines (figure 1) shows that this is not the case. In continuing the solution from the horizon along an anti-Stokes line, it is clear that the influence of transition point $t_{3}$ must be taken into account. The higher-order contributions are small because the integration contour encircling $t_{1}$ and $t_{2}$ in the BS formula can be taken in the region of the coordinate plane far away from $t_{3}$. This is exactly the case when our generalized formula can be used with confidence.

\section{Conclusions}

We have derived a generalization of the BS formula using the phase-integral method in its simplest form. The numerical results obtained from this formula are remarkably accurate even for large overtone index $n$. In this paper, the influence of three transition points, all considered as well separated from each other, is accounted for in the simplest possible way. Bearing this in mind, the numerical results obtained from the formula are surprisingly accurate. However, we expect the GBS formula to break down after some value of $n$ for several reasons, namely:

(i) For very high-overtone modes the anti-Stokes line spiraling towards the horizon runs close to the two transition points $t_{2}$ and $t_{3}$ several times. In the analysis of this situation one would have to take into account each turn of the spiral-see [10].

(ii) There are situations when $t_{2}$ and $t_{3}$ should be considered as close (when $|\operatorname{Im} \sigma| \approx|\operatorname{Re} \sigma|)$. This case has been considered by Andersson and Linnæus [10]. Their analysis yields reliable results in the intermediate regime where accurate results are not obtained from the BS formula or our GBS formula.

(iii) The increasing proximity of the fourth transition point to the three considered in this paper.

The success of our approach encourages further application of the phase-integral method to the cases of Reissner-Nordström and Kerr black holes. Beyond that, we want to investigate the possibility of applying the method to the interesting cases of neutron stars. However, our experience shows that the error estimates within the phase-integral method require a more careful consideration (see comments in [3] and [4]). This point is at present being investigated and the results will be discussed in a forthcoming paper.

\section{Acknowledgments}

NA thanks the Royal Society and The Royal Swedish Academy of Sciences for financial support. MEA wishes to thank Coordenaçăo de Aperfeiçoamento de Pessoal 
de Ensino Superior (CAPES) and the Royal Society for financial support during different periods while this work was being made, and the Department of Physics and Astronomy of the University of Wales College of Cardiff for its hospitality.

\section{References}

[1] Guinn J W, Will C M, Kojima Y and Schutz B F 1990 High-overtone normal modes of Schwarzschild black holes Class. Quantum Grav. 7 L47-L53

[2] Fröman N, Fröman P O, Andersson N and Hökback A 1992 Black-hole normal modes: phase-integral treatment Phys. Rev. D 45 2609-16

[3] Andersson N, Araújo M E and Schutz B F 1993 The phase-integral method and black-hole normal modes Class. Quantum Grov $10735-55$

[4] Araújo M E, Nicholson D and Schutz B F 1992 On the Bohr-Sommerfeld formula for black hole normal modes Preprint

[5] Leaver E W 1985 An analytic representation for the quasi-normal modes of Kerr black holes Proc. R. Soc. A 402 285-98

[6] Nollert H-P and Schmidt B G 1992 Quasinormal modes of Schwarzschild black holes: defined and calculated via Laplace transformation Phys. Rev. D 45 2617-27

[7] Andersson N 1992 A Numerically accurate investigation of black hole normal modes Proc. R. Soc. A 439 47-58

[8] Mathews J and Walker R L 1970 Mathematical Methods of Physics 2nd edn (Reading, MA: AddisonWesley)

[9] Thylwe K E 1983 Phase-integral formulae in the complex angular momentum (CAM) pole analysis J. Phys. A: Math. Gen. 16 3325-40

[10] Andersson N and Linnæus S 1992 Quasi-normal modes of a Schwarzschild black hole: improved phase-integral treatment Phys. Rev. D $464179-87$ 